\title{
Accessible Language: Foreign Language Teaching Strategies for Blind and Visually Impaired Students
}

\author{
Tatiana Belova \\ Russian State Specialized Arts Academy \\ Moscow, Russia \\ E-mail: beltatiana11@gmail.com
}

\begin{abstract}
This paper focuses on the question of the accessibility of foreign language teaching strategies, techniques and resources for students with visual impairment. The main teaching activities are examined from the point of view of their adaptation to the needs of visually impaired students in the mixed inclusive language classroom. The main purpose of this article is to explain the major difficulties blind and visually impaired students encounter in their language learning process and offer activities that are beneficial not only for students with sight loss but for all the other students in the group. The article is addressed to the foreign language teachers who are obliged to include blind or visually impaired students in their classes.
\end{abstract}

Keywords-Foreign language teaching; visually impaired; accessible language teaching; foreign language for visually impaired; alternative teaching strategies

\section{INTRODUCTION}

Learning foreign languages is important for everybody in our modern society. It becomes even more important for people with special educational needs, such as for visually impaired individuals. The lack of sight makes difficult for them not only to move around the city, to travel or to get a new job, sometimes it can create problems even in communication, especially when it comes to meeting people from other countries or getting acquainted with new places and cultures. Visually impaired and blind people can not see pictures, movies and images, body language, gestures and mimics are hardly understood as well. In this case speech remains for them the only means for communication in a new country. That's why learning a foreign language gives the visually impaired individuals a possibility to travel (even alone), to communicate (either in person or via internet), to find new friends and professional contacts. It gives them an opportunity to visit new places, to know new cultures and even to find new interesting jobs. Finally, learning a new language can help individuals with physical disabilities feel more confident. Thus, learning a language remains one of the most accessible and, natural tools for the visually impaired people that can be quite easily and naturally acquired. On the other hand, it becomes a very powerful tool for socialization and even for rehabilitation for individuals with special educational needs.
Since the word is the key element of communication for individuals with severe sight problems, it should become the key point in developing accessible foreign language teaching strategies for visually impaired students. But lots of our modern textbooks and teaching techniques are based on images, films, role cards, gestures and other visual aids that make commonly used teaching methods almost unavailable for people with complete or partial sight loss. That is why many blind language students prefer traditional methods of teaching based on translation and learning vocabulary. When I ask my blind and partially sighted students about their preferred methods of learning a language, most of them mention listening to the radio and pot casts, reading books, learning dialogues, doing grammar exercises, learning vocabulary and doing translation exercises. That means they prefer a non visual learning approach.

With the help of new technologies it becomes more and more possible to create new accessible foreign language teaching materials and adapt new accessible teaching strategies, such as online testing, doing online exercises and quizzes, chatting, correspondence and even using available software for modern smartphones. Moreover, very often adjustments the teachers make with the needs of the visually impaired students help other sighted peers in the group to develop better reading, writing, listening and speaking skills. This adjustments and small changes made by teachers often help the entire group to feel more relaxed and confident. On the other hand, if teachers feel anxious about including blind or visually impaired students in their traditional language classes, the anxiety is often understood by other group members and can cause discomfort or even create a negative classroom environment. In this paper the basic language skills are discussed with regard to the needs of students with sight problems.

\section{READING AND WRITING}

Some teachers believe that blind and visually impaired students can learn languages only by sound. They think that the best way for such students to learn words and grammar is to listen to the commercially available registered audio courses and repeat constantly after the speaker word by word and phrase by phrase. It is true, but only to a certain extend. For some students, for example for those who lost their sight in an older age and can not use computers or read Braille, it 
remains the only means for learning a new language. But as a teacher I'm strongly convinced that every student regardless of his / her abilities should try to read and, possibly, write, even in Braille or with the help of magnifying devices. If a student doesn't read, the perception of foreign language is corrupted. It is heard to learn languages only by sound. From my teaching experience I know that there are many students with well-developed auditory skills, but there are also a great number of learners, blind from birth or early childhood, whose auditory abilities are rather poor. They can not recognize speech in dialogues or answer oral questions. It means that not all students with visual impairment have good auditory skills and as teachers we should be attentive developing our adaptive teaching resources.

Very often blind students use tactile memory, which is quite strong and in many cases substitutes the visual memory of the majority of our students. That is why this powerful teaching tool can not be underestimated. Although blind students can not see and memorize pictures, they can and must read words and texts equally with their sighted peers. Reading by fingers as well as by eyes creates a certain image of the word in the memory and helps to memorize it better. That is why it is advisable for every teacher who has a "brail reader" in the class to prepare materials in Braille as well as in normal print. In alternative, a teacher can give to a visually impaired student all the needed materials in advance before the course so they could scan, print and prepare them. Otherwise blind students may not only get false perception of the language but sometimes also feel discriminated. The teacher should encourage blind and low vision students to read even if it takes time. It's worth knowing that some people with visual impairment don't read Braille. For this reason a teacher should find the way to present materials in an appropriate format. It can be a large print, an electronic material or an audio format.

It has been noticed that it becomes impossible for blind and low visual students to read texts in a normal structural way, like their sighted peers do. Braille texts are read by fingers, sign by sign. Blind people perceive information in a sequential linear way, which makes impossible several types of reading comprehension exercises. Some types of reading, such as skimming and scanning, may present difficulty as well. In reading tasks it is not easy for a blind person to find the needed information in the text, even if they have it in Braille or digital format. Especially, it becomes difficult with the time limit. Sometimes blind people have a good memory and after reading the text they try to memorize the passage in order to answer the comprehension questions after the text. As a result, they read rather slowly, often get tired quickly and sometimes lose concentration after reading. For that reason it is better to use short texts and change activities several times during the lesson. It is also advisable for teachers to give more time for reading to students with visual impairment. On the other hand, there are many activities that can be done successfully in a mixed group including visually impaired students. Here are only some of them: 1. Prediction. A teacher reads the title of the text and asks students to brainstorm the ideas about its contents. As an alternative, a teacher can give a list of words and expressions and ask the group to predict what they are going to read. 2. Role reading. It is good to read dialogues and polilogues, so a visually impaired person can make a pause and listen to his partners, then swich to reading. 3. Pair work. A teacher can ask each student to read a part of the text. Then, a visually impaired person can work with the sighted partner and exchange information about the passage they have read. 4. Retelling. It is quite easy for a person with visual impairment to retell the text, so this activity may be done without any adaptation. The students may be asked to retell the text from different points of view or from different characters. 5. Comprehension true / false or multiple choice questions can be also done without any additional adaptation. 6 . If the text is quite long, it is good to give it as a home task to prepare in advance. It is much easier for a blind person to read the familiar text and to work with it.

Usually, modern language textbooks have lots of graphic elements, such as columns, graphs, tables or pictures. Sometimes they accompany texts. All these elements should be correctly converted into Braille or digital format because they create huge difficulties for

Blind people and it is the task of the teacher to minimize them. Columns and tables can be converted to the plain text.

It has been noticed that students with low vision who use magnifiers read text in the same linear way. Their eyes get tired very quickly. That is why they often read slowly with lots of mistakes. In order to achieve good results, teachers should give them more time for doing reading tasks, make more pauses and change activities more frequently. Low vision students usually read printed materials. Some students can read normal print without any adaptations but the majority of students will need large print texts or magnifying devices. Many students with low vision have less stamina for reading texts. That is why sometimes it is recommended to reduce the amount of reading and have the student read shorter passages. On the other hand, some scholars recommend encouraging the student to read extensively in order to increase their reading speed and stamina. Recommendations are similar to those with sighted students. They include repeated readings, choral reading and echo reading.

Modern technologies are of great help to blind people and to their foreign language teachers. Sometimes there's almost no difference between blind and sighted students in the way they accomplish several tasks. When blind students "read" using screen reading programs and speech synthesizers, they listen to the text. But, actually, they listen in the same sequential and linear mode, which means they can listen word by word or letter by letter. They can return to the needed word or paragraph or check the spelling. That's why blind people call this process "reading", even if it is a particular kind of listening. This specific kind of reading as well as Braille helps them to perceive words and texts.

Writing assignments can be given to visually impaired students as their home task, so they can do them quietly at their own pace. They can write using their home computer and then sent the text to the teacher via e-mail or bring in the 
class. If the goal is to control writing in class, we can give the task in electronic format and the student can write using his / her personal laptop. If the student is not good at computers, the teacher can read the task to the student and the student should complete the task orally. At the exam an assistant can read the test to the blind learner and then record and write his or her answers. Generally, in Russian high schools visually impaired students work without assistants. It is a very good practice to do a pair work where students with and without visual impairment work together. They discuss the task together and then, the sighted student can write the answers down. It is advisable to give more pair work in the inclusive classes, where people with and without disabilities can socialize with different partners. It helps to overcome the anxiety that can sighted people have while meeting a visually impaired student for the first time. As a teacher I often use combined worksheets where the information is written in normal print or large print and in Braille. Usually, sighted students love such worksheets because they feel that they can work equally with visually impaired peers. Sometimes, it is worth making combined grammar schemes, where the information is presented in normal print or in large print and in Braille. Another available method is using tests. Both online tests and paper tests can be done quite easily by people with visual problems. Online tests are good for all types of students. Learners can do them at their own pace from home using their computer or tablet. Online tests vary according to the topic of the course. Teachers can use them for testing not only separate topics but the whole course as well. Nevertheless, it is strongly recommended to test the accessibility of all the online resources. They should meet all the criteria of the accessibility for people that use screen reading software.

\section{LISTENING AND SPEAKING}

Since listening and speaking are two main activities that visually impaired people will use in their daily life and social interaction, it is very important to develop good listening skills with visually impaired students, even if these skills were poorly developed previously. Moreover, it results very useful for other sighted peers who are used to rely mainly on their visual memory. In order to develop good listening skills we can use the following techniques and activities in the mixed ability groups:

Word chains. Ask student to make a sentence using one word from the unit, and then ask the second student to repeat the previous one and add their own word. Continue till somebody gets wrong. The variety of this activity is called "I'm going on a trip". The teacher starts by saying "I'm going on a trip and I'll take an umbrella with me", then students continue adding their own elements.

Piece of music. The teacher can play 30 second music pieces of different genres and ask students what picture they imagine while listening.

Counting syllables. Give students a list of words to remember. Ask them to read 3 words out loud. Ask the second student to repeat these words and to clap out the number of syllables. You can then increase the number of words up to 10 or more and ask students to repeat all the words and clap out all the syllables.

Biography. Good for memorizing numbers. Read to the student the name, the surname, the occupation of a person and his telephone number and ask him to repeat this information without writing it down. For example, Maria Gabriella Sanches, housewife, 331-10-97. We can add the information we need for our curriculum, such as countries, nationalities, hobbies and other things.

Snail talk. Provide the list of words to the class then tell them that you are going to pronounce one word very slowly. THE first student to guess the word wins and chooses a new word.

Intonations. Ask students to pronounce the needed phrase with the intonation he wants. Ask the second student to repeat the phrase using a different intonation. Students can use different phrase stress, voice tones and intonations. The task is to listen carefully in order not to repeat the intonation that has been already used.

Complete the sentence. The teacher reads the sentence without one word. Then, asks the students to guess this word and repeat the whole sentence.

Word positions. A teacher pronounces a sequence of words like February may, July, September, April. Then, asks the student to repeat the second, the fourth, and the fifth word in the sequence. The teacher can extend the number of words up to 10 or more, depending on the ability of the group.

Memorizing facts. Ask the students at the end of each lesson what they were doing during the lesson. Ask them to recall all the activities done from the beginning of the lesson to the end or starting from the end of the lesson to the beginning.

The message. The exercise can be done in a small group of $4-6$ students. Ask all students except one to go out of the classroom. Read a message to a student containing names, dates or time. Then invite the second student. The first student should tell the message to the second one without changing it. Compare the initial message with the one the last student says.

Number dictation. Read a sequence of numbers to a student in the foreign language then ask a student to translate them into Russian without asking to repeat. Read a sequence of numbers in Russian language and ask another student to translate them into foreign language. To increase the difficulty, it is possible to give numbers in Russian and in foreign language together and ask the student to translate tem vice versa.

Sometimes listening tasks presented in our modern textbooks may result difficult for blind students, even if they have well-developed listening skills. If they use their computer with screen reading software, it is impossible to listen both to a speech synthesizer and to the recording at the same time in order to answer the question or fill in the gaps. Blind students get distracted because they should follow the speaker and listen to their synthesizer, which is almost 
impossible. The only way of completing these tasks for a visually impaired student is to read all the questions first and hold them in memory, which is not easy at all. That's why it is advisable to give extra time for such activities. It is good to do them in pairs. If pair work is not possible, we should give the visually impaired student to listen to the recording individually, with pauses if needed. If this task is given at the exam, visually impaired students should be well prepared and trained enough in order to feel less stress. It is better to avoid such tasks as filling in the gaps into the texts with the words heard on the CD because it is not easy for the visually impaired student to read the gapped text while listening. The activities with pictures should be well-prepared as well. As a variant, a teacher may ask other students in the group to describe all the visual material presented in the task. If students work in pairs sighted peer may be asked to describe the picture to his blind partner.

Speaking may seem the easiest activity to practice with students with visual impairment. All the students can speak. Every student can participate in discussions, role plays or dialogues. On the other hand, some modern textbooks have such tasks like describing pictures, which can be done in a slightly different way. The best way to deal with pictures is make students working in pairs. A blind student should ask questions about the picture, where his sighted partner answers the questions and they make a short dialogue or invent a story, based on the pictures. If we give role cards, a teacher or a peer may read what is written on the card.

It is important to use tactile books and pictures working with children. But for older students it makes almost no sense. Blind people consider all imagery information useless and forget it quickly if it is not connected with their practical experience. The majority of information exists in the form of signs or words. That is why it is important to explain from word to possible image, not the other way round. The thinking process of blind individuals is connected with concrete or abstract concepts. In comparison with sighted peers, blind people can not think using images. All image communication has to be translated into spoken or written words. In any case, teachers should explain everything written on the blackboard. It's the task of the teacher convert into spoken words all the visual content.

Videos and pictures. It is important to be careful using films and videos. In order to do this it the teacher needs to build the atmosphere of mutual help and respect. In this case "watching" films together as well as "describing" pictures together with a sighted peer can result in a huge benefit for both students not only for language purposes but also for understanding each other's personalities. It is impossible to ask visually impaired students to watch the whole film or a part of it in order to answer comprehension questions. Sometimes films in foreign languages can be very difficult to understand without the visual content. For this reason all long video passages should be presented with audio description. Teachers can describe the visual content to the student or even better they can ask other students to describe it. Sometimes we can ask students to work in pairs. Sighted partners can concentrate on what they see, while blind partners concentrate on what they hear. After that students exchange information. Another way to deal with the videos is to watch video spots, which are quite short and can be easily described by the group. Teachers can ask visually impaired students to watch the video in advance before the lesson; in this case it will be easier for both a teacher and a student to work with this material.

There's a common prejudice that blind students can be offended when it comes to using pictures. On the contrary, I noticed that activities with photos may be very fun and relaxing. For example, sighted students describe the photos, while blind peers ask questions to understand the description. It means that the description should be very clear and detailed.

Booklets. I usually have booklets designed for my students and use them over and over again in various groups. In booklets the information is presented in a clear and comprehensive way. Booklets can be given to students in all preferred formats, including digital or audio formats, large print or Braille.

\section{VOCABULARY AND GRAMMAR}

In our modern society people learn languages for different purposes. One of the most common aims of learning the language is traveling and getting to know new cultures. Visually impaired and blind people also like traveling. They can travel with their sighted relatives or friends, make business trips, visit conferences or seminars abroad, participate in various social and cultural events. For all these reasons they need foreign languages. But what language blind people use in standard traveling situations? The main point is that usually blind people need to know more words and expressions than their sighted friends. If a sighted person enters a café or a shop, he can look at the counters and get an idea of what to have. While blind persons should always ask a question in order to understand what they can buy. After that they should listen to the answer and make a dialogue. If a sighted person needs to take a train he can look at the timetable, while a blind person should listen carefully to announcements or ask for help. In all these situations blind people need the knowledge of the language and especially of the vocabulary. For this reason in our classes we can practice various communicative situations. Some grammar and vocabulary exercises presented in our textbooks, such as matching exercises or scrambled words may also create slight difficulties for visually impaired students. We can modify these exercises and easily substitute them with different ones.

\section{CONCLUSION}

In our modern society many teachers are obliged to deal with students with a variety of professional or special educational needs, including students with physical disabilities. The knowledge about accessible foreign language techniques and strategies can help teachers and instructors to reduce anxiety and to feel more confident in the class. Here are some basic points to consider planning a foreign language course for students with severe sight problems: 
- Prepare materials in electronic format, which makes it easier for students with special needs to adapt the information in the suitable format: large print, electronic format, Braille or audio.

- Make the materials and textbooks of the course available in advance, so there is enough time for visually impaired students to prepare them in a suitable format.

- Think beforehand how to verbalize everything you are doing in the class. Think how to switch to a verbal style of communication.

- Read out loud what is written on the blackboard, describe or ask other peers to describe all pictures and other visual content.

- Inform the visually impaired student if you plan to use videos or films, discuss beforehand alternative ways to present it. If needed give the video to a student before the lesson so he can watch it at home and ask somebody to describe it.

- Don't give essays with hand-written comments. Usually it is not possible for students with sight problems to read a hand written text. In this case give a feedback to a student orally.

- Don't overcompensate preparing your adaptive materials. It is better to maintain balance so everybody in the group can feel safe and confident.

Finally, it is worth mentioning that working with people with special needs it is important to create a particular class atmosphere based on trust, mutual respect, cooperation and comfort. In this situation peer support becomes very important. Teaching students to interact with each other should become a top priority in inclusive language classes. In this case learning process means feeling safe and learning to work together with other peers. It means learning things in several different ways and applying them in practice in various situations. Planning our language courses we need to remember that often slight changes may be very significant and beneficial not only for students with disabilities but to every student in our inclusive group.

\section{REFERENCES}

[1] Broome C. Insight without sight. // CBM. V. 25: Psychology of blindness. - AEBC, 2007. - p. 31 - 36

[2] Conroy P. Total Physical Response: An Instructional Strategy for Second -Language Learners who are visually impaired. // Journal of Visual Impairment and Blindness, 1999, 93 no 5. - pp. 315 - 318.

[3] Guinan Holly. ESL for students with visual impairments. // Journal of Visual Impairment and Blindness, 1997, 91 no 6. - pp. 555 - 563.

[4] Lewin-Jones, J. and Hodgson, J. Differentiation strategies relating to the inclusion of a student with severe visual impairment in higher education (modern foreign languages). // British Journal of Visual Impairment, 2004. 22/1. - pp. 32 - 36

[5] Richardson,J. \& Roy, A. The representation and attainment of students with a visual impairment in higher education. // British Journal of Visual Impairment, 2002. 20/1
[6] Solntseva L.I. Tiflopsychology of childhood. - M, Poligraph service, 2000. - $140 \mathrm{p}$. 